\title{
Spontaneous ureteral rupture after extracorporeal shock wave lithotripsy: a case with review of literature
}

\begin{abstract}
Objective: To study the diagnosis and treatment of spontaneous ureteral rupture after extracorporeal shock wave lithotripsy (ESWL).

Methods: A case of spontaneous ureteral rupture after ESWL was analyzed retrospectively. A 30-year-old male presented to the emergency clinic with severe and progressive right flank pain for the last 24 hours. He did not complain of frequent urination, painful urination, hematuria, fever, dysuria. He underwent ESWL of right renal stone 4 days ago. Physical examination showed tenderness in the right abdomen and percussion pain in the right renal area. His leukocyte count and neutrophils and lymphocytes were $22.36^{*} 10^{9} / \mathrm{L}$, $92.0 \%, 6.3 \%$, respectively; blood urea nitrogen and blood creatinine were $9.0 \mathrm{mmol} / \mathrm{L}$, $166 \mathrm{umol} / \mathrm{L}$ respectively. His ultrasound was suggestive of right hydronephrosis and right distal ureteral stones and a small perinephric fluid. No improvement, treated by a medical expulsive therapy for 2 days. CTU (Computed Tomography Urography) showed the right upper ureteral rupture and right hydronephrosis and right lower ureteral stones. Holmium laser lithotripsy under ureteroscope was performed under general anesthesia and double J stent was inserted.

Results: The operation was a success. His routine blood and serum creatinine returned to normal 6 days later. Another day later, CTU showed less right hydronephrosis than before and no perinephric fluid and no ureteral stones. His catheter was removed 10 days later and was discharged without any complications. The double-J stent was removed 6 weeks later.

Conclusion: Spontaneous ureteral rupture after ESWL is a rare entity. It can cause serious complications, including urinoma, kidney damage, sepsis. A careful clinical and ultrasound or CTU monitoring with a high index of suspicion of it is necessary after each ESWL therapy. The prompt treatment of the condition will achieve good results.
\end{abstract}

Keywords: ESWL, spontaneous ureteral rupture, double-j stent, ureteral stones, frequent urination, painful urination, hematuria, fever, dysuria, urinoma, kidney damage, sepsis
Volume 7 Issue 5 - 2019

\section{Zhang Junwei, Peng Dongtao, Wang Gang, Xu Min, Tang Shijie}

Department of Urology, Affiliated Hospital of Southwest University, China

Correspondence: Zhang Junwei, Department of Urology, Affiliated Hospital of Southwest University, Chongqing 400700 , China, Tel +8613983392। 47 ,

Email 845454677@qq.com, junweizhang97@gmail.com

Received: September 11, 2019 | Published: October 18, 2019
Abbreviations: ESWL, extracorporeal shock wave lithotripsy; CTU, computed tomography urography

\section{Introduction}

ESWL is safer, less invasive, less painful, less costly and faster in recovery than operation in the treatment of kidney and upper ureteral stones. It is a good fit for the outpatient treatment and preferred for ureteral stones less than $1.5 \mathrm{~cm}$ and renal stones less than 2 cm. ${ }^{1}$ Spontaneous ureteral rupture is rare, usually thought to occur following an obstructive pathology. ${ }^{2}$ There has been only one reported case of spontaneous ureteral rupture after ESWL. ${ }^{3}$ We report a rare case of spontaneous ureteric rupture in a patient with a clinical history of right renal stones treated with ESWL and review the literature report as follows.

\section{Case presentation}

A 30 years old gentleman was admitted to the emergency clinic of our institution with a 1 day history of a right-sided severe and progressive flank pain. He did not complain of frequent urination, painful urination, hematuria, fever, and dysuria. He had ESWL for right kidney stones four days ago. Physical examination showed tenderness in the right abdomen and obvious percussion pain in the right renal area. His leukocyte count, neutrophils, and lymphocytes were $22.36 * 10^{9} / \mathrm{L}, 92.0 \%, 6.3 \%$, respectively; blood urea nitrogen is $9.0 \mathrm{mmol} / \mathrm{L}$ and blood creatinine $166 \mathrm{umol} / \mathrm{L}$. His urine analysis, serum liver function tests, serum amylase/lipase as well as all other biochemical tests were within normal limits.

Ultrasonography of urinary system showed hydronephrosis of right kidney, calculus of right distal ureter and small perirenal effusion. Ultrasonography of digestive system showed no abnormality of liver, pancreas and spleen. There was no improvement, treated by a medical expulsive therapy for 2 days. A subsequent CTU (Computed Tomography Urography) scan showed right hydronephrosi and right lower ureteral stones 2-11 mm (Figure 1). A vast shadow of water with $\mathrm{CT}$ value about 2-10 HU is around the right kidney and upper ureter (Figure 2). The ureteral rupture with leakage of contrast is shown from the proximal ureter at the level of the $3^{\text {rd }}$ lumbar vertebra.

With general anesthesia, the patient was promptly taken up for the right ureteroscopic holmium laser lithotripsy and placement of a double-J stent (4.7Fr). Intraoperatively, we found 4 stones (diameter: 
$0.2-0.4 \mathrm{~cm}$ ) in Right ureter bladder wall segment. After holmium laser lithotripsy, we found that there was a $1.0 \mathrm{~cm}$ diameter gap at the ureteropelvic junction with a little fatty tissue under it. Without stone residues insight, a double-J stent was positioned successfully after placement of a stiff guidewire. A $16 \mathrm{Fr}$ catheter was inserted to avoid ureteral reflux. There was no evidence of intra- and postoperative complications. His routine blood and serum creatinine returned to normal 6 days after operation.

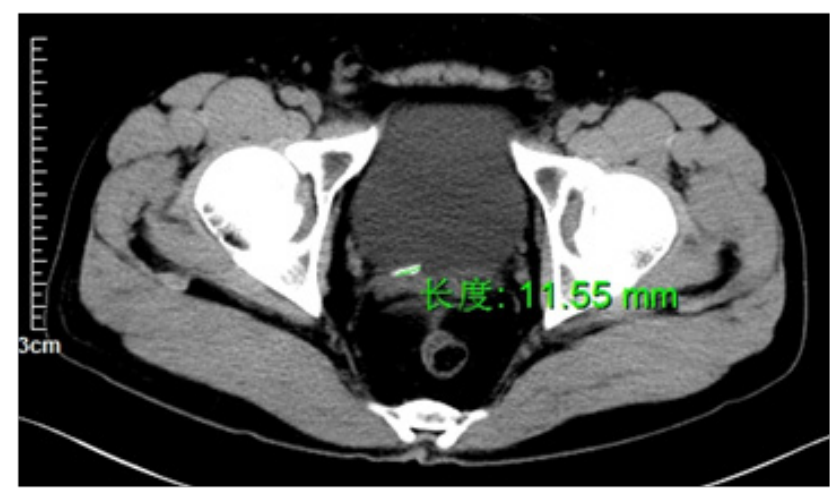

Figure I Right lower ureteral stones 2-I I mm.

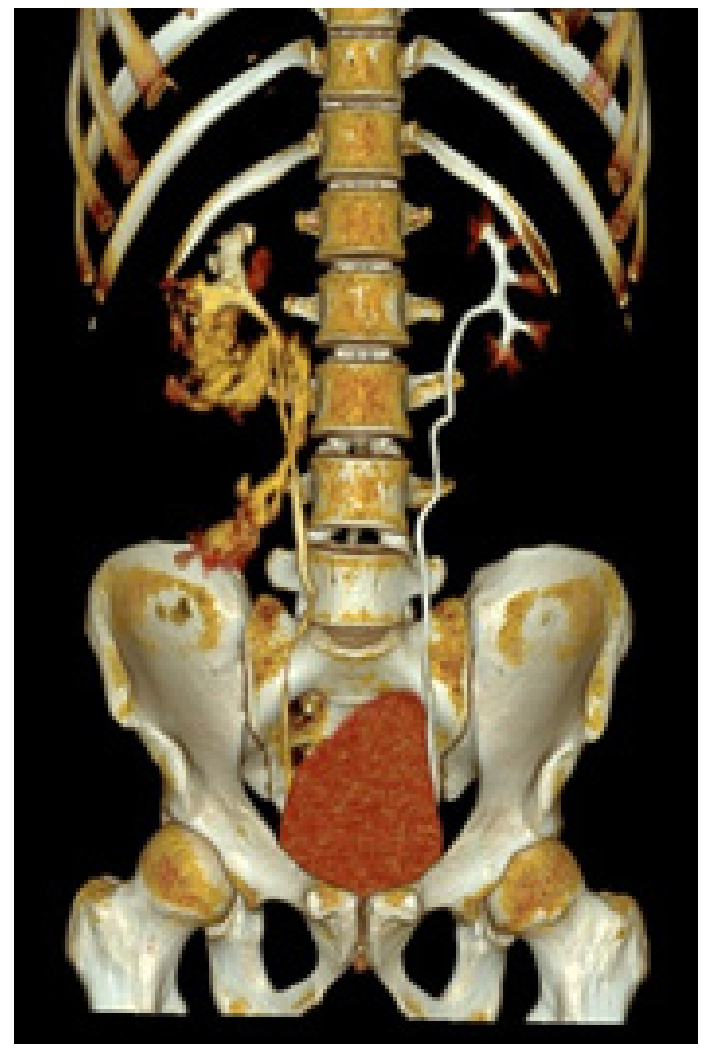

Figure $\mathbf{2}$ The ureteral rupture with leakage of contrast from the proximal ureter.

One day after surgery, CTU showed less right hydronephrosis than before, no perinephric space effusion or ureteral stones, and correct placement of both proximal and distal extremities of the double-J stent. In order to fully drain and facilitate the healing of ureteral, the catheter was removed 10 days after operation. He was discharged without any complications. According to the experience of open surgery, double-J stent are usually retained for 4 to 8 weeks. The double-J stent was removed 6 weeks after the operation. After 6 months and 12 months, no abnormal findings were found by ultrasonography.

\section{Discussion}

ESWL is a relatively safe and effective modality and a widely used in the treatment of urinary stones. However, it could lead to renal hematoma, urinoma, retroperitoneal abscess, renal contusion, sepsis in some cases. ${ }^{4}$ Serious complications are rare but do occur with adjacent organ injury, like colonic perforation, pancreatitis, splenic rupture. ${ }^{5,6,7}$ Spontaneous ureteral rupture is even rarer especially after ESWL. ${ }^{3}$ Spontaneous ureteral rupture is by definition an extravasation of urine from the ureter without previous surgery, ureteric manipulation and external trauma of the ureter. ${ }^{8,910}$ The pathogenesis is unclear, no plausible explanation has been published in the literature. It is generally believed that urinary tract obstruction leads to ureteral lumen pressure, or a downward moving stones cause ulceration of the ureteral wall. ${ }^{11,12}$ Causative factors include urinary calculi, tumor, idiopathic retroperitoneal fibrosis, pregnancy, connective tissue diseases and urinary retention..$^{13,14}$

After urine extravasation, spontaneous ureteral rupture can cause peritoneal irritation, characterized by acute abdominal pain. Most don't have urinary tract symptoms or abnormal urine. It can cause series of problems, including retroperitoneal urinoma, urosepsis, infection, and subsequent renal impairment. ${ }^{15,16} \mathrm{It}$ is often misdiagnosed as other conditions, such as appendicitis or diverticulitis. Because history taking and examination can be unreliable to diagnose spontaneous ureteral rupture, a high index of suspicion after ESWL is maintained and further investigations should be utilized. In our case, the ultrasound was first performed and the patient was treated with a 2-day decompression treatment to promote stone discharge. His symptoms got worse, so we underwent CTU and found a rupture of the ureter. In our opinion, the downward moving stones after ESWL formed the lower ureteral stones and induced the lower uterus obstruction, which leads to upper ureteral lumen pressure and finally resulted in ureteral rupture. The patient underwent the right ureteroscopic holmium laser lithotripsy and inserted a double-J stent. He was discharged 10 days after the operation without any complications.

It is difficult to diagnose spontaneous ureteral rupture, so urologists should be aware of its occurrence after ESWL therapy. CTU is the right choice of technique in the diagnosis. After confirmation, treatment can be individualized based on aetiology. Typically, minimally invasive endoscopic procedures can successfully place a double-J stent tube into the renal pelvis. In conclusion, ESWL is a relatively safe and effective modality to treat urinary lithiasis. Spontaneous ureter rupture after ESWL could happen, although it is rare with only one previous reported case. It can cause urinoma, urosepsis, infection, and subsequent renal impairment. A careful clinical monitoring with a high index of suspicion of spontaneous ureter rupture is necessary after each ESWL therapy. CTU is a quick and accurate imaging modality to diagnose its possibility. Ureteric double-J stent is the most commonly used management option and the prompt treatment always achieve good results.

\section{Acknowledgments}

None. 


\section{Funding details}

None.

\section{Conflicts of interest}

The author declares there is no conflict of interest.

\section{Reference}

1. Türk C, Petř́k A, Sarica K, et al. EAU Guidelines on interventional treatment for urolithiasis. Eur Urol. 2016;69(3):475-482.

2. Torricelli FC, Danilovic A, Vicentini FC, et al. Extracorporeal shock wave lithotripsy in the treatment of renal and ureteral stones. Rev Assoc Med Bras. 2015;61(1):65-71.

3. Chen GH, Hsiao PJ, Chang YH, et al. Spontaneous ureteral rupture and review of the literature. Am J Emerg Med. 2014;32(7):772-774.

4. Searvance K, Jackson J, Schenkman N. Spontaneous Perforation of the UPJ: A Case Report and Review of the Literature. Urol Case Rep. 2016;26(10):30-32.

5. Kurz W, Klein B, Rumstadt B. Colonic perforation after extracorporeal shock wave lithotripsy. Dtsch Med Wochenschr. 2009;134(9):401-403.

6. White WM, Morris SA, Klein FA, et al. Splenic rupture following shock wave lithotripsy. Can J Urol. 2008;15(4):4196-4199.

7. Pace K, Spiteri K, German K. Spontaneous proximal ureteric rupture secondary to ureterolithiasis. J Surg Case Rep. 2016;11:1-3.

8. Liu S, Lin J, Huang C, et al. Spontaneous rupture of the ureter mimicking acute appendicitis: Two case reports. J Acute Med. 2011;1(2):61-63.
9. Choi SK, Lee S, Kim S, et al. A rare case of upper ureter rupture: Ureteral perforation caused by urinary retention. Korean J Urol. 2012;53(2):131133.

10. Sarmah PB, Noah A, Kelly B, et al. Asymptomatic ureteral rupture secondary to chronic urinary retention from massive prostatic enlargement. J Surg Case Rep. 2015;2015(11):pii: rjv135.

11. Ikeda S, Ishikawa M, Kato T. Spontaneous ureteral rupture during concurrent chemoradiotherapy in a woman with uterine cervical cancer. Gynecol Oncol Rep. 2015;13:18-19.

12. Inahara M, Kojima S, Takei $\mathrm{K}$, et al. Two cases of spontaneous rupture of upper urinary tract caused by the primary ureteral or renal pelvic tumor:a case report. Hinyokika Kiyo. 2009;55(1 ):31-34.

13. Ay D, Yencilek E, Celikmen MF, et al. Spontaneous rupture of ureter: an unusual cause of acute abdominal pain. Am J Emerg Med. 2012;30(2):390-392.

14. Pampana E, Altobelli S, Morini M, et al. Spontaneous ureteral rupture diagnosis and treatment. Case Rep Radiol. 2013;2013:851859.

15. Turgut M, Can C, Yenilmez A, Akcar N. Perforation of the upper ureter: a rare complication of extracorporeal shock wave lithotripsy. Urol Res. 2007;35(4):215-258.

16. Chen $\mathrm{CC}$, Chang $\mathrm{CH}$, Liu YL, et al. A tiny stone induced ureteral rupture. Urolithiasis. Ann Acad Med Singapore. 2010;39(12):948-942.

17. Eken A, Akbas T, Arpaci T. Spontaneous rupture of the ureter. Singapore Med J. 2015;56(2):29-31. 